


\title{
Calidad de la evidencia científica: un reflejo del estado actual de la Enfermedad de Chagas
}

\author{
Jefferson Antonio Buendía Rodríguez. MD, MSc en Epidemiología, MSc en Farmacología. \\ Instituto de Salud Pública. \\ Universidad Nacional de Colombia.
}

En Latinoamérica y especialmente en nuestro país, gran parte de la enfermedad cardiovascular se debe al la enfermedad de Chagas (ECHA); el resultado de la infección humana por Trypanosoma cruzi, descrita desde principios del siglo anterior. Según la OMS, 16-18 millones de personas en Latinoamérica están infectadas y otras 100 millones están en riesgo de adquirir la infección. Las consecuencias de esta enfermedad, que aparecen en la tercera o cuarta década de la vida de aquellos sujetos infectados (principalmente durante la infancia y en áreas rurales), son incurables por el momento y explican la pérdida de 2.740.000 DALYs (Disability Adjusted Life Years) en el continente; cuatro veces mas que la carga de enfermedad debida a malaria, lepra y leishmaniasis. En Colombia, país clasificado por la OPS como de activa transmisión de la enfermedad, se estima que 990.000 personas están infectadas mientras que 3.000.000 estarían en riesgo.

Es evidente la discordancia entre la carga de la ECHA, en términos de morbimortalidad, y el conocimiento generado para evitar la enfermedad; esto se refleja en la ubicación actual de la ECHA, entre las tres enfermedades más abandonadas del mundo. En ausencia de vacunación, quimioprofilaxis o tratamiento especifico, la única estrategia de control ha sido el control vectorial; estrategia que en países del cono sur ha logrado disminuir la transmisión, pero que en nuestro medio no ha podido tener el impacto esperado debido a los problemas de pobreza, violencia, migración interna y no mejoramiento de las condiciones de vivienda en áreas de transmisión activa. En síntesis, la ECHA es un problema de salud publica en nuestro país y el continente, donde los problemas de la región no han podido reducir la carga debida a esta enfermedad, debilitando las estrategias de control.

La valoración sistemática de la calidad de evidencia científica es una herramienta útil para la toma de decisiones no solo en el ambiente clínico sino en el momento de hacer políticas públicas. Diferentes métodos han sido diseñados para este objetivo. Sin embargo, el mas utilizado es la valoración de la evidencia según el diseño utilizado en los estudios primarios y la ausencia de sesgos en los mismos mediante escalas que ponderan la calidad metodológica en niveles de evidencia y grados de recomendación. Recientemente, el Ministerio de Protección Social y la Universidad Nacional de Colombia realizaron la actualización de la guía de diagnostico y tratamiento de la Enfermedad de Chagas planteadas en la resolución 412 del 2002 utilizando los métodos anteriormente mencionados para la construcción de esta guía clínica. Durante este proceso fue evidente la baja calidad de la evidencia existente en diagnostico y 
tratamiento en esta enfermedad. Problemas en los estudios diagnósticos como la utilización de muestreos inadecuados y sesgos de verificación, explican la heterogeneidad en los estimados de sensibilidad y especificidad; haciendo que las recomendaciones actuales de diagnósticos se fundamenten mas en consensos de expertos (nivel de evidencia 4, grado de recomendación D) que en estudios primarios.

Así mismo, la evidencia acumulada sobre la eficacia del tratamiento tripanomicida en pacientes con infección crónica por T. cruzi en términos de desenlaces clínicos, es limitada en cantidad y calidad. Aunque los resultados en desenlaces "sustitutos" (marcadores de carga parasitaria) son convincentes, los resultados en desenlaces clínicos son inciertos: no significativos estadísticamente, y heterogéneos. Los estudios que asocian los marcadores de carga parasitaria y desenlaces clínicos corresponden a estudios de cohorte con problemas metodológicos, como sesgo de selección y clasificación, que limitan su validez interna y con ello el nivel de evidencia y grado de recomendación que se puede derivar de los mismos. En síntesis, la evidencia actual solo permite la recomendación de tratamiento tripanomicida en pacientes con infección crónica por $T$. cruzi con el propósito de reducir la carga parasitaria pero no para mejorar su pronóstico clínico.

En conclusión, el panorama actual de la ECHA en nuestra región, es producto de la interacción entre la aplicación de estrategias de control limitadas -que desconocen la variabilidad socioeconómica y conflictos existentes en cada país - la falta de investigación científica con adecuada calidad metodológica que sea orientada al paciente, y los problemas de equidad que evitan el mejoramiento las condiciones socioeconómicas y educativas en áreas rurales de transmisión activa de la enfermedad. Es este un llamado a la comunidad científica para que desde allí se genere una sensibilización de la problemática social en esta enfermedad y se redireccione la generación de evidencia en la Enfermedad de Chagas.

\section{Referencias.}

1. Chagas C. Nova Tripanosomiase humana. Estudios sobre a morfolojia e o ciclo evolutivo do Schizotrypanum cruzi., n sp., agente etiologico de nova entidad morbida do homem. Mem Inst Oswaldo Cruz 1909; 1: 159-218.

2. WHO Division of Control of Tropical Diseases. Chagas Disease Elimination. Burden and Trends. WHO web page. www.who.int/ $\mathrm{ctd} / \mathrm{html} / \mathrm{chagburtre} \cdot \mathrm{html}$.

3. Murray C, Lopez A. The Global Burden of Disease. Cambridge Harvard University Presss, 1996.

4. Schmunis G. American tripanosomiasis as a public health problem. In: "Chagas' disease and the nervous system". 3-29. PAHO. Washington DC 1994.

5. PAHO. Health Conditions in the Americas. Volume I. Washington DC, 1990.

6. Yamei U, The world's most neglected diseases. BMJ 2002; 325:176-177.

7. Villar J. Commentary: Control of Chagas' disease: let's put people before vectors. Int J Epidemiol. 2001 Aug;30(4):894-5.

8. Orozco L, Camargo D. Evaluación de tecnologías diagnosticas y tipos de muestreos Biomédica 1997; 17:321-324.

9. Villar J, Schapachnik E, Freilij H, Altech J. Tratamiento de la enfermedad de Chagas ("treatment of Chagas' disease"). In: Doval HC, Tajer CD (eds) Evidencias en cardiología 3. GEDIC. Buenos Aires 2003. 
